\title{
Prevalence of Tobacco Habits and Attitude toward Its Cessation among Outpatients of Mahatma Gandhi Mission's Dental College and Hospital, Navi Mumbai, Maharashtra
}

\author{
Juveria Syed Ali Hussain, Sabita M Ram
}

\begin{abstract}
Background: Information regarding the tobacco habits in patients visiting Mahatma Gandhi Mission's (MGM) Dental College and Hospital is scarce. Therefore, development and better implementation of the tobacco cessation initiatives can be undertaken with the knowledge of the tobacco use among patients.
\end{abstract}

Materials and methods: A cross-sectional, questionnairebased study was conducted among the outpatients of MGM Dental College and Hospital from July to September, 2012. Questions regarding the form of tobacco used, frequency, duration and attitude toward stopping its consumption were asked. Statistical analysis was carried out using descriptive statistics and chi-square tests using Statistical Software package (SPSS) version 17.

Results: $314 / 505(62.1 \%)$ of the patients reported of tobacco use. Majority 162/314 (51.6\%) of the patients used smokeless tobacco in which mishri and gutkha were mostly consumed. Work pressure was the common 100/314 (31.8\%) cause of tobacco use. More than half the patients had tried to quit the habit and showed awareness toward its adverse effects on oral and general health.

Conclusion: Tobacco use has become an important concern due to its growing addiction among all age groups and gender. It is imperative to understand the psychosocial ideology and pattern of tobacco use among the patients in order to effectively counsel and motivate them to stop the habit.

Keywords: Tobacco, Smoking, Habits.

How to cite this article: Hussain JSA, Ram SM. Prevalence of Tobacco Habits and Attitude toward Its Cessation among Outpatients of Mahatma Gandhi Mission's Dental College and Hospital, Navi Mumbai, Maharashtra. J Contemp Dent 2013; 3(2):57-60.

Source of support: Nil

Conflict of interest: None declared

\section{INTRODUCTION}

Globally, tobacco is the leading preventable cause of death which causes one person to die every 6 seconds and it is estimated to rise to more than 8 million deaths per year by 2030 of which more than $80 \%$ will live in low- and middleincome countries. ${ }^{1}$ WHO sources emphasizes the rate of tobacco consumption especially in developing countries like India as an epidemic. ${ }^{2}$ The availability and the increasing consumption of variety of smoked and smokeless products makes tobacco epidemic in India a significant public health concern. Bidi smoking is a very common form of smoked tobacco, and smokeless tobacco forms include pan, mishri, pan masala, gutkha, khaini and areca nut.

India's tobacco consumption is the second largest in the world after China. ${ }^{3}$ An estimated 1 million Indians die annually from tobacco-caused diseases, and projections forecast that by 2020 , tobacco will account for $13 \%$ of deaths in India. ${ }^{4}$ Implementations of educational interventions and tobacco cessation programs are a necessity and have been successful in controlling the tobacco epidemic. However, it is important to have information regarding the tobacco use status of the particular region so as to initiate a program against tobacco use. Also, health professionals play an imperative role in persuading patients to stop tobacco habits as awareness toward adverse effects of tobacco use and willingness to quit influence the patient's attitude to stop tobacco-related habits.

Limited data about tobacco-associated habits in the patients visiting the Mahatma Gandhi Mission's (MGM) Dental College and Hospital, Navi Mumbai, India, was available and no data regarding attitude toward tobacco use cessation was present. For better implementation of the tobacco intervention program, the prevalence of tobacco use among the dental patients needs to be described. The aim of the study was to evaluate the prevalence of tobacco habits in patients visiting MGM Dental College and Hospital and their perception and attitude toward the quitting tobacco use. The objectives of the study were as follows:

1. To assess the demographic profile and the prevalence of tobacco habits in patients visiting MGM Dental Hospital.

2. To assess patients attitude toward tobacco control and their willingness to quit tobacco use.

\section{MATERIALS AND METHODS}

\section{Study Design, Instrument and Data Collection}

A cross-sectional study was conducted from July 2012 to September 2012, among the outpatients of MGM Dental College and Hospital. The proposal, including ethical views, was approved by the ethics committee. The study instrument was a 3-page, 20-item, structured questionnaire which was an adapted version of the Global Adult Tobacco Survey (GATS) questionnaire. ${ }^{5}$ The questionnaire was available in three languages—English, Hindi and Marathi-depending 
on the preference of the respondent. Patients who were unable to provide consent or unable to speak English, Hindi or Marathi in order to answer the questionnaire were not included in the study. The respondents were informed about the purpose of the study and asked to voluntary participate in the study. A written consent was obtained and confidentiality of their information was assured. The topics covered in the questionnaire were demographic profile of the patients, use of tobacco, role of health care professionals and patient's attitude toward tobacco control and awareness of harmful effects of tobacco. A pilot study was carried out to check the validity of the questionnaire (Cronbach's alpha <0.5).

Current tobacco users (smoke/smokeless tobacco) were defined as those who smoked or chewed tobacco presently and the frequency of tobacco use was defined as daily (at least once per day) or weekly (at least once a week) or occasional (at least once per week). Nontobacco users were those who did not currently smoke or chew tobacco.

\section{Statistical Analysis}

The analysis was performed using statistical package for social sciences, SPSS, version 17. Frequency tables, descriptive statistics and graphs were used to present the data. Further statistical analysis was done using chi-square test for association between attributes. A p-value of $<0.05$ was considered as statistically significant.

\section{RESULTS}

\section{Sociodemographic Characteristics}

The study population comprised of 600 patients, out of which 505 (84.1\%) patients completed the questionnaire. Among the 505 respondents, 314 (62.1\%) gave a history of tobacco use either in the smoke or smokeless or both forms. As shown in Table 1, out of 314 tobacco users, 225 (71.7\%) of the patients who were males and 89 (28.3\%) were females and majority $170 / 314$ (54.1\%) of them were between the age group of 25 to 44 . The occupational status showed that employees (government/self-employed) reported with the highest tobacco use followed by housewives and students and 106/314 (33.7\%) had not received schooling or did not complete primary school (Table 1 ).

\section{Prevalence and Habits of Currents Tobacco Users}

Overall 314/505 (62.1\%) patients reported of tobacco use, out of which 110 (35\%) were only smokers (cigarette, bidi or both) and 162 (51.6\%) were users of smokeless tobacco. 142/314 (13.4\%) respondents used tobacco in both forms. Many of the respondents used multiple products of smoke and smokeless forms of tobacco and more than half 172/314 (54.8\%) were daily tobacco users. 106/314 (33.8\%) of the respondents had been habitual to tobacco usage since the last 5 years followed by 70/314 (22.3\%) had started it in the last 6 months. Work pressure was reported by majority $100 / 314$ (31.8\%) of the tobacco users followed by stress relieve and peer pressure (Table 2).

\section{Tobacco Awareness and Attitude among Patients toward Tobacco Control}

Among the 314 tobacco users, 204 (65\%) had tried to quit tobacco use and majority of them had tried to stop its use in the past 1 year. 105/314 (33.4\%) had never tried to stop tobacco use. Only 31/314 (9.9\%) patients were willing to quit tobacco use from the same day with 260/314 (82.8\%)

Table 1: Sociodemographic characteristics of current tobacco users

\begin{tabular}{lrr} 
1. Gender & & \\
- Male & 225 & 71.7 \\
- Female & 89 & 28.3 \\
2. Age group (in years) & & \\
- 18-24 & 89 & 28.3 \\
- 25-44 & 170 & 54.1 \\
- 45-64 & 46 & 14.6 \\
- >65 & 9 & 2.9 \\
3. Marital status & & \\
- Single & 154 & 49.0 \\
- Married & 18 & 5.7 \\
- Divorced/separated/widowed & 142 & 45.2 \\
4. Occupational status & & \\
- Student & 56 & 17.8 \\
- Employee (government/ & 173 & 55.0 \\
- self-employed) & & \\
- Housewife & 63 & 20.1 \\
- Retired & 13 & 4.1 \\
- Unemployed & 9 & 2.8 \\
5. Education & & \\
- Primary school & 42 & 13.3 \\
- Secondary school & 86 & 27.3 \\
- Higher secondary and above & 80 & 25.4 \\
- No schooling or did not complete & 106 & 33.7 \\
$\quad$ primary school & & \\
\hline
\end{tabular}

\section{Table 2: Characteristics of tobacco use}

\begin{tabular}{lrr} 
Variable & $N$ & Percentage \\
\hline 1. Form of tobacco use & & \\
- Only smokers & 110 & 35.0 \\
- Only smokeless & 162 & 51.6 \\
- Both forms & 142 & 13.4 \\
2. Frequency of tobacco use & & \\
- Daily & 172 & 54.8 \\
- Weekly & 48 & 15.3 \\
- Occasionally & 94 & 29.9 \\
3. Reason for tobacco use & & \\
- Stress relieve & 56 & 17.8 \\
- Work pressure & 100 & 31.8 \\
- Fun & 51 & 16.2 \\
- Peer pressure & 77 & 24.5 \\
- Other & 30 & 9.6 \\
\hline
\end{tabular}


did believe that tobacco causes serious illness and 291/314 (92.7\%) had seen warnings on tobacco products.

\section{DISCUSSION}

The International Classification of Diseases (ICD-10) has recognized that 'tobacco dependence is a disease'. ${ }^{2}$ In developing countries like India wherein tobacco consumption is rising by $3.4 \%$ per year, it is the most common cause of head and neck cancers. ${ }^{6}$ Our study showed that among the $62.1 \%$ of the tobacco users, majority were males which is consistent with other studies. ${ }^{7-9}$ With cultural diversity and ethnicity, even today it is a social norm that men predominantly smoke and are habitual to use of tobacco whereas it is not favorable for women or young girls to smoke which leads to use of smokeless form of tobacco among them. In our study, these habits were observed mostly between the age group of 25 and 44 years followed by 18 to 24 years and least among those more than 65 years which is similar to a study in Illinois among hospital patients which showed that the peak age for smoking was at 35 to 54 years with lower rates of smoking at either extreme of age. $^{8}$ Also, information on marital status revealed that $154 / 314$ (49\%) of the tobacco users were single followed by 142/314 (45.2\%) married with 173/314 (55\%) of the users being employed and 63/314 (20.1\%) were housewives. The use of tobacco among the younger age group and working class can be attributed to financial, social as well as psychological factors. The educational status revealed that about $33.7 \%$ of the tobacco users were illiterate or had not completed their primary schooling followed by $27.3 \%$ who had completed only secondary school. These results were found to be statistically significant $(\mathrm{p}<0.001)$. It has been observed that preference for smokeless form of tobacco is inversely related to education and income. ${ }^{10}$ Similarly, a study in Turkey showed that most of the smokers had completed only 5 years of primary education. ${ }^{11} \mathrm{~A}$ casecontrol study on patients in Kerala demonstrated that nontobacco users were more educated as compared to tobacco users. ${ }^{12}$ Thus, the root cause of prevalence of tobacco habits is lack of education which subsequently leads to financial burden and employment hassles.

In the present study, tobacco habits showed that smokeless form of tobacco was commonly used followed by smoking and both forms. The consumption rate of mishri and gutkha was found to be higher as compared to other products. Smoking habits revealed that bidi smoking was more common as compared to smoking cigarettes. The type of tobacco use differs from region to region. Mishri is prepared by roasting tobacco until it is uniformly black and powdered and then applied on the gums with fingers for relieving toothache and can be related to its higher use as compared to gutkha. Bidi is a handmade smoking stick made by rolling a dried, rectangular piece of temburni leaf with sun-dried, flaked tobacco filled into a conical shape and the roll is secured with a thread. Gutkha is a mixture of areca nut, tobacco and some condiments, marketed in different flavors in colorful pouches. The low cost and ease of availability of the smokeless products makes its use so high in these areas. In the state of Maharashtra, smokeless tobacco use is more common than smoking. ${ }^{13}$ All these products are known to have deleterious effects on oral and general health of the individual. This has considerable public health implication as it is known that people change over from smokeless form to smoking over a period of time. In a study from the large metropolitan city of Mumbai, the prevalence of tobacco use was highest for smokeless form among both women and smoked form among men followed by $10 \%$ who used both forms. It is a general perception that chewing of these products is considered less harmful than smoking and use of smokeless forms of tobacco eventually leads to smoking. According to the data reported by the Cancer Patients Aid Association of India in 2004, it was observed that bidi smoking with a prevalence of $40 \%$ was more common as compared to cigarettes with a prevalence of $20 \%$ smoking of cigarettes followed by the use of smokeless tobacco in various forms (prevalence of $40 \%) .{ }^{14}$ Artificial sweeteners and flavors are added to these commercial products of smokeless tobacco which makes them more appealing and also neutralize the unpleasant taste of tobacco.

The frequency of tobacco use was found to be high with daily consumption being reported by majority of the tobacco users followed by occasional and weekly use. Reasons for tobacco habits were highest for work, stress and peer pressure (Table 2). Peer pressure was also reported by $46 \%$ of the tobacco users as the reason for tobacco use in a study in the state of Kerala. ${ }^{9}$ It has been concluded that smoking is a significant predictor of financial stress. ${ }^{15}$ Most of the users were addicted to tobacco within 30 minutes of waking up which indicates the tobacco dependence of these patients. Nicotine is known to have a dual effect as it acts both a stimulant and depressant and it is likely that its effect is determined by the mood of the user, parental habits, the environment, and the circumstances. In the present study, it was noticed that among the total 314 tobacco users, about 106/314 (33.8\%) were in this habit for less than 5 years and 75/314 (23.9\%) for more than 5 years. Tobacco use was carried out in the presence of family members in 207/314 (65.9\%) of the patients which is an area of concern as parent and relatives have an influential effect on a child's habits and behavior. It has been found among the Indian studies that the mean age of initiation of tobacco use varies from 8 to 15 years. ${ }^{16,17}$ Worldwide, tobacco users first tried tobacco starting as early as 10 to 18 years. ${ }^{18,19}$ 
It was found in our study that 204/314 (65\%) of the patients had tried to quit the habit of smoking and/or chewing tobacco with 103/314 (32.8\%) having tried in the past 1 year which is similar to other study. ${ }^{7}$ Only $31 / 314$ (9.9\%) of the tobacco users showed readiness to quit from the same day. A study showed that $61 \%$ of the patients showed willingness to quit who smoked. ${ }^{8}$ In our study, it was observed that 291/314 (92.7\%) were aware that tobacco is harmful and 260/314 (82.8\%) had seen the statutory warnings on tobacco packets. A total of $57 \%$ of the patients were aware of the ill effects of tobacco consumption in a dental institute in India. ${ }^{20} 291 / 314$ (92.7\%) of the patients had seen warnings on packets of tobacco products which indicates that despite most of the patients visiting the hospital demonstrated addiction to tobacco use, there was general awareness of the harmful effects of tobacco. However, there was lack of firmness in the attitude of the patients to stop this habit which led them to resume its use. Therefore, strong initiative and educational interventions are imperative to eliminate these habits among all age groups. Also, it is the collective responsibility of the dental students and professionals to counsel patients on the adverse effects of tobacco use and help them quit its habit.

One of the limitations of this study is the potential of recall bias and under-reporting by the patients as the study was based on self-reporting of tobacco habits. The habits reported in the questionnaire may also differ from the actual smoking or tobacco use pattern. The study is based on a convenient sample and therefore not a representative of the Indian population. Therefore, additional research needs to be followed up to encourage patients to quit tobacco use and attention needs to be given to increase the importance of education among the patients in order to reduce the economic and social burden related to unemployment. It is also essential to assess the effectiveness of educational initiatives undertaken to persuade patients to stop tobacco use by future studies.

\section{REFERENCES}

1. World Health Organization. WHO report on the global tobacco epidemic, 2009. Geneva: World Health Organization; 2008: 1-342.

2. Reddy KS, Gupta PC. Report on tobacco control in India. Mumbai: Ministry of Health and Family Welfare; 2004:1-397.

3. Shimkhada R, Peabody JW. Tobacco control in India. Bull World Health Organ 2003;81(1):48-52.

4. Keluskar V, Kale A. An epidemiological study for evaluation of oral precancerous lesions, conditions and oral cancer among Belgaum population with tobacco habits. Biosci Biotech Res Comm 2010 Jun;3(1):50-54.
5. Global adult tobacco survey collaborative group. Global adult tobacco survey (GATS): Core Questionnaire with Optional Questions, Version 2.0. Atlanta, GA: Centers for Disease Control and Prevention; 2010 Nov;1-66.

6. World Health Organization. International Statistical Classification of Diseases and Related Health Problems. 10th ed. Geneva: World Health Organization; 1994 URL: http://apps.who.int/ classifications/apps/icd/icd10online2003/fr-icd.htm accessed on Sept 25, 2012.

7. Keluskar VK, Kale AD, Ammanagi RA, Hebbal MI, Janwad PC. Chewing patterns of areca nut, betel quid, and tobacco among patients reporting to a Dental Institute in India. Oral Oncol 2011 Jul;47(1):S43.

8. Katz A, Goldberg D, Smith J, Trick W. Tobacco, alcohol and drug use among hospital patients: Concurrent use and willingness to change. J Hosp Med 2008 Sep;3(5):369-375.

9. Vellappally S, Jacob V, Smejkalová J, Shriharsha P, Kumar V, Fiala Z. Tobacco habits and oral health status in selected Indian population. Cent Eur J Public Health 2008 Jun;16(2):77-84.

10. Gupta PC. Survey of sociodemographic characteristics of tobacco use among 99, 598 individuals in Bombay, India, using handheld computers. Tob Control 1996 Summer;5(2):114-120.

11. Cakmak G, Saglam ZA, Saler T, Yenigun M, Temiz LU. Socioeconomic status of smokers resenting to the outpatient service: does chronic obstructive pulmonary disease make a difference? Indian J Chest Dis Allied Sci 2012 Jan-Mar;54(1):19-22.

12. Balagopal PG, George NA, Venugopal A, Mathew A, Ahamed IM, Sebastian P. Tobacco related habits among first degree relatives of patients undergoing surgery for advanced head and neck malignancies in India. Asian Pacific J Cancer Prev 2012; 13(1);217-220.

13. Gupta PC, Ray CS. Smokeless tobacco and health in India and South Asia. Respirology 2003 Dec;8(4):419-431.

14. Chaly PE. Tobacco control in India. Indian J Dent Res 2007 Jan-Mar;18(1):2-5.

15. Siahpush M, Borland R, Scollo M. Smoking and financial stress. Tob Control 2003 Mar;12(1):60-66.

16. Kapil U, Goindi G, Singh V, Kaur S, Singh P. Consumption of tobacco, alcohol and betel leaf amongst school children in Delhi. Indian J Pediatr 2005 Nov;72(11):993.

17. Chadda RK, Sengupta SN. Tobacco use by Indian adolescents. Tob Induc Dis 2003;1(1):111-119.

18. Reddy KS, Arora M. Tobacco use among children in India: a burgeoning epidemic. Indian Pediatr 2005;42:757-761.

19. Ahmed NU, Ahmed NS, Semenya KA, Elzey JD, Larson C, Bennett CR, Hinds JE. Prevalence and correlates of initiation of smoking behavior among preteen black and white children. J Natl Med Assoc 2004 Feb;96(2):200-208.

\section{ABOUT THE AUTHORS}

\section{Juveria Syed Ali Hussain (Corresponding Author)}

General Dentist, Department of Prosthodontics, Mahatma Gandhi Mission's Dental College and Hospital, Navi Mumbai, Maharashtra India, e-mail: juveriaali@yahoo.com

\section{Sabita M Ram}

Dean, Professor and Head, Department of Prosthodontics, MGM Dental College and Hospital, Navi Mumbai, Maharashtra, India 